\title{
A NEW RESULT ON AVERAGING THEORY FOR A CLASS OF DISCONTINUOUS PLANAR DIFFERENTIAL SYSTEMS WITH APPLICATIONS
}

\author{
JACKSON ITIKAWA ${ }^{1}$, JAUME LLIBRE$^{1}$ AND DOUGLAS D. NOVAES ${ }^{2}$
}

\begin{abstract}
We develop the averaging theory at any order for computing the periodic solutions of discontinuous piecewise differential system of the form

$$
r^{\prime}= \begin{cases}F^{+}(\theta, r, \varepsilon) & \text { if } \quad 0 \leq \theta \leq \alpha \\ F^{-}(\theta, r, \varepsilon) & \text { if } \quad \alpha \leq \theta \leq 2 \pi,\end{cases}
$$

where $F^{ \pm}(\theta, r, \varepsilon)=\sum_{i=1}^{k} \varepsilon^{i} F_{i}^{ \pm}(\theta, r)+\varepsilon^{k+1} R^{ \pm}(\theta, r, \varepsilon)$ with $\theta \in \mathbb{S}^{1}$ and $r \in D$, where $\mathrm{D}$ is an open interval of $\mathbb{R}^{+}$, and $\epsilon$ is a small real parameter.

Applying this theory, we provide lower bounds for the maximum number of limit cycles that bifurcate from the origin of quartic polynomial differential systems of the form $\dot{x}=-y+x p(x, y), \quad \dot{y}=x+y p(x, y)$, with $p(x, y)$ a polynomial of degree 3 without constant term, when they are perturbed, either inside the class of all continuous quartic polynomial differential systems, or inside the class of all discontinuous piecewise quartic polynomial differential systems with two zones separated by the straight line $y=0$.
\end{abstract}

\section{Introduction and Statement of the Main Results}

One of the main open problems in the qualitative theory of polynomial differential systems in $\mathbb{R}^{2}$ is the determination of their limit cycles, see for instance [10]. Bifurcations of limit cycles have been exhaustively studied in the last century and is closely related to the Hilbert's $16^{\text {th }}$ problem. However, in spite of all efforts, up to now there is no general method to solve this question.

Bifurcation of limit cycles in continuous planar differential systems are still largely studied. Nonetheless, due to the considerable number of discontinuous phenomena in the real world, see for example [5, 20] and the references therein, a significant interest in the investigation of limit cycles of discontinuous piecewise differential systems has arisen. For instance in [17], applying the theory of regularization, the averaging theory is extended up to order 1 for studying the

Corresponding author Douglas D. Novaes: Departamento de Matemática, Universidade Estadual de Campinas, Rua Sérgio Baruque de Holanda, 651, Cidade Universitária Zeferino Vaz, 13083-859, Campinas, SP, Brazil, email: ddnovaes@ime.unicamp.br

2010 Mathematics Subject Classification. Primary 34C07, 34C29, 34C25, 37G15.

Key words and phrases. periodic solution, averaging method, non-smooth differential system, discontinuous differential system, uniform isochronous center. 
periodic solutions of systems of the form $x^{\prime}=\varepsilon(F(t, x, \varepsilon)+\operatorname{sign}(h(x)) G(t, x, \varepsilon))$. In [14] there is a version of the averaging theorem up to order 2 for a bigger class of discontinuous piecewise differential equations $x^{\prime}=\varepsilon F_{1}(t, x, \varepsilon)$. Finally in [15] it is stated averaging theorems for studying the periodic solutions of discontinuous piecewise differential equations of the form $x^{\prime}=F_{0}(t, x)+\varepsilon F_{1}(t, x, \varepsilon)$.

Our main goal in this paper is to develop the averaging theory at any order for a particular class of discontinuous piecewise differential systems, see subsection 1.1. Then, as an application of this theory, we present in subsection 1.2 the bifurcation of limit cycles of quartic polynomial differential systems of the form $\dot{x}=-y+x p(x, y), \quad \dot{y}=x+y p(x, y)$ when they are perturbed, either inside the class of all continuous quartic polynomial differential systems, or inside the class of all discontinuous piecewise quartic polynomial differential systems with two zones separated by the straight line $y=0$. Here $p(x, y)$ is a polynomial of degree 3 without constant term.

\subsection{Results in averaging theory}

We develop the averaging theory at any order for computing the periodic solutions of discontinuous piecewise differential system of the form

$$
r^{\prime}=\left\{\begin{array}{lll}
F^{+}(\theta, r, \varepsilon) & \text { if } \quad 0 \leq \alpha \\
F^{-}(\theta, r, \varepsilon) & \text { if } \quad \alpha \leq 2 \pi
\end{array}\right.
$$

where

$$
F^{ \pm}(\theta, r, \varepsilon)=\sum_{i=1}^{k} \varepsilon^{i} F_{i}^{ \pm}(\theta, r)+\varepsilon^{k+1} R^{ \pm}(\theta, r, \varepsilon) .
$$

The set of discontinuity of system (1) is $\Sigma=\{\theta=0\} \cup\{\theta=\alpha\}$ if $0<\alpha<2 \pi$. Here $F_{i}^{ \pm}: \mathbb{S}^{1} \times D \rightarrow \mathbb{R}$ for $i=0,1, \ldots, n$, and $R^{ \pm}: \mathbb{S}^{1} \times D \times\left(-\varepsilon_{0}, \varepsilon_{0}\right) \rightarrow \mathbb{R}$ are $\mathcal{C}^{k+1}$ functions, where $D$ is an open and bounded interval of $(0, \infty)$, and $\mathbb{S}^{1} \equiv \mathbb{R} /(2 \pi)$.

We point out that taking $\alpha=2 \pi$ system (1) becomes continuous. So the averaging theory developed in this section also applies to continuous differential systems.

For $i=1,2, \ldots, k$, we define the averaged function $f_{i}: D \rightarrow \mathbb{R}$ of order $i$ as

$$
f_{i}(\rho)=\frac{y_{i}^{+}(\alpha, \rho)-y_{i}^{-}(\alpha-2 \pi, \rho)}{i !}
$$

where $y_{i}^{ \pm}: \mathbb{S}^{1} \times D \rightarrow \mathbb{R}$, for $i=1,2, \ldots, k-1$, are defined recurrently as

$$
\begin{aligned}
y_{i}^{ \pm}(\theta, \rho)= & i ! \int_{0}^{\theta}\left(F_{i}^{ \pm}(\phi, \rho)+\sum_{l=1}^{i} \sum_{S_{l}} \frac{1}{b_{1} ! b_{2} ! 2 ! b_{2} \cdots b_{l} ! l ! b_{l}} .\right. \\
& \left.\partial^{L} F_{i-l}^{ \pm}(\phi, \rho) \prod_{j=1}^{l} y_{j}^{ \pm}(\phi, \rho)^{b_{j}}\right) d \phi,
\end{aligned}
$$


where $S_{l}$ is the set of all $l$-tuples of non-negative integers $\left(b_{1}, b_{2}, \cdots, b_{l}\right)$ satisfying $b_{1}+2 b_{2}+\cdots+l b_{l}=l$, and $L=b_{1}+b_{2}+\cdots+b_{l}$.

As we shall see the averaged functions $f_{i}$ control the existence of isolated periodic solutions of system (1). Since these functions are obtained directly from $y_{i}^{ \pm}$using (3), we give in the Appendix the explicit formulae of $y_{i}^{ \pm}$'s up to $i=7$.

Our main result on the periodic solutions of (1) is the following.

Theorem 1. Assume that, for some $\ell \in\{1,2, \ldots, k\}, f_{i}=0$ for $i=1,2, \ldots, \ell-1$ and $f_{\ell} \neq 0$. If there exists $\rho^{*} \in D$ such that $f_{\ell}\left(\rho^{*}\right)=0$ and $f_{\ell}^{\prime}\left(\rho^{*}\right) \neq 0$, then for $|\varepsilon|>0$ sufficiently small there exists a $2 \pi$-periodic solution $r(\theta, \varepsilon)$ of (1) such that $r(0, \varepsilon) \rightarrow \rho^{*}$ when $\varepsilon \rightarrow 0$.

Theorem 1 is proved in section 2 .

\subsection{Periodic solutions in planar differential quartic systems with a uniform isochronous center-focus type singular point}

The study of bifurcation of limit cycles in planar analytic differential systems which have a center-focus equilibrium at the origin and whose angular speed is constant has increased in the last decades. In such systems, the origin is the unique finite singular point and if it is a center, it will be a uniform isochronous one. These systems, up to a linear change of coordinates, may be written as

$$
\dot{x}=-y+x H(x, y), \quad \dot{y}=x+y H(x, y),
$$

where $H$ is an analytic function and $H(0,0)=0$.

The relevance of investigating these systems is due, on the one hand, to their importance in the general problem of isochronicity. Indeed, any analytic system with linear part $(-y, x)^{t}$ has an isochronous center at the origin if and only if it is possible to be transformed, by applying the following analytic change of coordinates $(x, y) \rightarrow\left(x+P\left(y^{2}\right), y+Q(x, y)\right)$ in system (4), for more details see $[18,1]$. On the other hand, system (4) in polar coordinates $x=r \cos \theta, y=r \sin \theta$ is written as

$$
\dot{r}=\sum_{k \geq 1} H_{k}(\cos \theta, \sin \theta) r^{k+1}, \quad \dot{\theta}=1,
$$

where each $H_{k}$ is the homogeneous part of degree $k$ of the function $H$. These systems can be written under the form of an Abel generalized differential equation

$$
\frac{d r}{d \theta}=\sum_{k \geq 1} H_{k}(\cos \theta, \sin \theta) r^{k+1} .
$$

Equation (5) provides information about system (4), and vice versa, since the constant solution $r=0$ of (5) corresponds to the origin of (4), and the periodic solutions of (5) with $r>0$ correspond to periodic orbits of (4), see [3, 1]. 
The bifurcation of limit cycles in planar differential polynomial systems of the form (4), that is, polynomial systems of degree $n$ of the form

$$
\dot{x}=-y+x p(x, y), \quad \dot{y}=x+y p(x, y),
$$

where $p(x, y)$ is a polynomial in $x$ and $y$ of degree $n-1$ and $p(0,0)=0$, has been intensively studied, see for instance $[6,8,9]$ and the bibliography therein.

Consider a planar differential polynomial system and $q \in \mathbb{R}^{2}$ a singular point of this system. We say that $q$ is a weak focus if it is a center for the linearized system at $q$.

Let $q \in \mathbb{R}^{2}$ be a center of a differential polynomial system in $\mathbb{R}^{2}$, without loss of generality we can assume that $q$ is the origin of coordinates. We say that $q$ is an isochronous center if it is a center having a neighborhood such that all the periodic orbits in this neighborhood have the same period. We say that $q$ is a uniform isochronous center if the system, in polar coordinates $x=r \cos \theta$, $y=r \sin \theta$, takes the form $\dot{r}=G(\theta, r), \dot{\theta}=k, k \in \mathbb{R} \backslash\{0\}$, for more details see Conti [8]. The next result is well-known and a proof for it can be found in [12].

Proposition 2. Assume that a planar differential polynomial system $\dot{x}=P(x, y)$, $\dot{y}=Q(x, y)$ of degree $n$ has a center at the origin of coordinates. Then, this center is uniform isochronous if and only if by doing a linear change of variables and a rescaling of time it can be written under the form (6).

Algaba, Reyes, Ortega and Bravo [2], in 1999, and Chavarriga, García and Giné [7], in 2001, independently provided the following characterization of nonhomogeneous quartic polynomial systems with an isolated uniform isochronous center at the origin.

Theorem 3. Consider $p(x, y)=\sum_{i=1}^{3} g_{i}(x, y)$ where $g_{i}(x, y)$ for $i=1,2,3$ are homogeneous polynomials of degree $i, g_{1}^{2}+g_{2}^{2} \neq 0$ and $g_{3} \neq 0$ such that (6) is a quartic polynomial differential system. Then system (6) has a uniform isochronous center at the origin if and only if it is reversible. In this case, modulo a rotation and a rescaling of the independent variable, system (6) can be written as

$$
\begin{aligned}
& \dot{x}=-y+x\left(A_{1} x+B_{2} x y+C_{1} x^{3}+C_{3} x y^{2}\right), \\
& \dot{y}=x+y\left(A_{1} x+B_{2} x y+C_{1} x^{3}+C_{3} x y^{2}\right) .
\end{aligned}
$$

where $A_{1}, B_{2}, C_{1}, C_{3} \in \mathbb{R}$.

In the case of homogeneous uniform isochronous centers, Conti [8] proved the following theorem in 1994.

Theorem 4. Let $p(x, y)=\sum_{i+j=n-1} g_{i, j} x^{i} y^{j}$ be a homogeneous polynomial of degree $n-1$. Then system (6) has a uniform isochronous center at the origin if either $n$ is even, or if $n$ is odd and 


$$
\sum_{\nu=0}^{n-1}\left[g_{n-1-\nu, \nu} \int_{0}^{2 \pi} \cos ^{n-1-\nu} \theta \sin ^{\nu} \theta d \theta\right]=0 .
$$

By Theorem 4 the homogeneous quartic polynomial systems of the form (6) always have a uniform isochronous center at the origin.

A classification of the global phase portraits of the planar quartic polynomial differential systems of the form (7) is provided in [12].

We study the limit cycles that bifurcate from the origin of the planar differential quartic polynomial systems of the form (6).

More precisely, let $H_{c}(n)$ denote the maximum number of limit cycles that bifurcate from the origin of system (6), when it is perturbed inside the class of all continuous polynomial differential systems of degree $n$, and $H_{d}(n)$ denotes the maximum number of limit cycles that bifurcate from the origin of system (6), when it is perturbed inside the class of all discontinuous piecewise polynomial differential systems of degree $n$ with two zones separated by the straight line $y=0$. We provide lower bounds for $H_{c}(4)$ and $H_{d}(4)$ in both cases when the origin is either a uniform isochronous center, or a weak focus. The method used for obtaining these lower bounds is based on the averaging theory.

This work extends previous results in [11], where we studied the bifurcation of limit cycles in cubic polynomial differential systems of the form (6).

In order to prove our results we also need the Descartes Theorem about the number of zeros of a real polynomial, see [4].

Theorem 5 (Descartes theorem). Consider the real polynomial $r(x)=a_{i_{1}} x^{i_{1}}+$ $a_{i_{2}} x^{i_{2}}+\ldots+a_{i_{r}} x^{i_{r}}$ with $0=i_{1}<i_{2}<\ldots<i_{r}$ and $a_{i_{j}} \neq 0$ real constants for $j \in\{1,2, \ldots, r\}$. When $a_{i_{j}} a_{i_{j+1}}<0$, we say that $a_{i_{j}}$ and $a_{i_{j+1}}$ have a variation of sign. If the number of variations of signs is $m$, then $r(x)$ has at most $m$ positive real roots. Moreover, it is always possible to choose the coefficients of $r(x)$ in such a way that $r(x)$ has exactly $r-1$ positive real roots.

We consider the following family of continuous systems

$$
\begin{aligned}
& \dot{x}=-y+x p(x, y)+\sum_{i=1}^{4} \varepsilon^{i} p_{i}(x, y), \\
& \dot{y}=x+y p(x, y)+\sum_{i=1}^{4} \varepsilon^{i} q_{i}(x, y),
\end{aligned}
$$


where

$$
\begin{aligned}
p_{j}= & \alpha_{0}^{j}+\alpha_{1}^{j} x+\alpha_{2}^{j} y+\alpha_{3}^{j} x^{2}+\alpha_{4}^{j} x y+\alpha_{5}^{j} y^{2}+\alpha_{6}^{j} x^{3}+\alpha_{7}^{j} x^{2} y+\alpha_{8}^{j} x y^{2}+\alpha_{9}^{j} y^{3} \\
& +\alpha_{10}^{j} x^{4}+\alpha_{11}^{j} x^{3} y+\alpha_{12}^{j} x^{2} y^{2}+\alpha_{13}^{j} x y^{3}+\alpha_{14}^{j} y^{4}, \\
q_{j}= & \beta_{0}^{j}+\beta_{1}^{j} x+\beta_{2}^{j} y+\beta_{3}^{j} x^{2}+\beta_{4}^{j} x y+\beta_{5}^{j} y^{2}+\beta_{6}^{j} x^{3}+\beta_{7}^{j} x^{2} y+\beta_{8}^{j} x y^{2}+\beta_{9}^{j} y^{3} \\
& +\beta_{10}^{j} x^{4}+\beta_{11}^{j} x^{3} y+\beta_{12}^{j} x^{2} y^{2}+\beta_{13}^{j} x y^{3}+\beta_{14}^{j} y^{4},
\end{aligned}
$$

and the discontinuous systems

$$
\left(\begin{array}{c}
\dot{x} \\
\dot{y}
\end{array}\right)=\mathcal{X}(x, y)=\left\{\begin{array}{lll}
X_{1}(x, y) & \text { if } y>0 \\
X_{2}(x, y) & \text { if } y<0
\end{array}\right.
$$

where

$$
\begin{gathered}
X_{1}(x, y)=\left(\begin{array}{c}
-y+x p(x, y)+\sum_{i=1}^{k} \varepsilon^{i} p_{i}(x, y) \\
x+y p(x, y)+\sum_{i=1}^{k} \varepsilon^{i} q_{i}(x, y)
\end{array}\right) \\
X_{2}(x, y)=\left(\begin{array}{c}
-y+x p(x, y)+\sum_{i=1}^{k} \varepsilon^{i} u_{i}(x, y) \\
x+y p(x, y)+\sum_{i=1}^{k} \varepsilon^{i} v_{i}(x, y)
\end{array}\right) \\
u_{j}=\gamma_{0}^{j}+\gamma_{1}^{j} x+\gamma_{2}^{j} y+\gamma_{3}^{j} x^{2}+\gamma_{4}^{j} x y+\gamma_{5}^{j} y^{2}+\gamma_{6}^{j} x^{3}+\gamma_{7}^{j} x^{2} y+\gamma_{8}^{j} x y^{2}+\gamma_{9}^{j} y^{3} \\
+\gamma_{10}^{j} x^{4}+\gamma_{11}^{j} x^{3} y+\gamma_{12}^{j} x^{2} y^{2}+\gamma_{13}^{j} x y^{3}+\gamma_{14}^{j} y^{4}, \\
v_{j}=\delta_{0}^{j}+\delta_{1}^{j} x+\delta_{2}^{j} y+\delta_{3}^{j} x^{2}+\delta_{4}^{j} x y+\delta_{5}^{j} y^{2}+\delta_{6}^{j} x^{3}+\delta_{7}^{j} x^{2} y+\delta_{8}^{j} x y^{2}+\delta_{9}^{j} y^{3} \\
+\alpha_{10}^{j} x^{4}+\delta_{11}^{j} x^{3} y+\delta_{12}^{j} x^{2} y^{2}+\delta_{13}^{j} x y^{3}+\delta_{14}^{j} y^{4},
\end{gathered}
$$

and $k=4$ or $k=7$. In both cases, the continuous and the discontinuous one we have to consider either

(10) $p(x, y)=t_{10} x+t_{01} y+t_{20} x^{2}+t_{11} x y+t_{02} y^{2}+t_{30} x^{3}+t_{21} x^{2} y+t_{12} x y^{2}+t_{03} y^{3}$, with $t_{i j} \in \mathbb{R}, i+j=1,2,3, t_{30}^{2}+t_{21}^{2}+t_{12}^{2}+t_{03}^{2} \neq 0$, or

$$
p(x, y)=t_{10} x+t_{11} x y+t_{30} x^{3}+t_{12} x y^{2},
$$

with $t_{30}^{2}+t_{12}^{2} \neq 0$, or

$$
p(x, y)=t_{30} x^{3}+t_{21} x^{2} y+t_{12} x y^{2}+t_{03} y^{3} .
$$

In the following we state our results.

Theorem 6. Using averaging theory of order 4 we obtain, for $|\varepsilon| \neq 0$ sufficiently small, $H_{d}(4) \geq 6$ for the differential system (9) with $p(x, y)$ of the form (10) (i.e. system (9) has a weak focus or a uniform isochronous center at the origin).

Theorem 6 is proved in section 3 .

Theorem 7. Using averaging theory of order 4 we obtain, for $|\varepsilon| \neq 0$ sufficiently small, $H_{d}(4) \geq 5$ for the differential system (9) with $p(x, y)$ either of the form (11) or (12) (i.e. system (9) has a uniform isochronous center at the origin). 
Theorem 7 is proved in section 4 .

Theorem 8. Using the averaging theory of order 7 we obtain, for $|\varepsilon| \neq 0$ sufficiently small, $H_{d}(4) \geq 6$ for the differential system (9) with $p(x, y)$ of the form (11) and $\alpha_{0}^{j}=\beta_{0}^{j}=\gamma_{0}^{j}=\delta_{0}^{j}=0, j=1, \ldots, 7$.

Theorem 8 is proved in section 5 .

Theorem 9. Using the averaging theory of order 4 we obtain, for $|\varepsilon| \neq 0$ sufficiently small, $H_{c}(4) \geq 2$ for the differential system (8) with $p(x, y)$ of the form (10).

Theorem 10. Using the averaging theory of order 4 we obtain, for $|\varepsilon| \neq 0$ sufficiently small, $H_{c}(4) \geq 1$ for the differential system (8) with $p(x, y)$ either of the form (11) or (12).

Theorems 9 and 10 are proved in section 6 .

To prove Theorems 6 and 7 (respectively Theorems 9 and 10) we shall use the averaging theory of order 4 for discontinuous (respectively continuous) differential systems, together with a rescaling of the variables. In these proofs we can see, using Descartes Theorem, that the lower bounds which appear in the theorems are actually upper bounds for the averaging theory of order 4, too. Then, from the proofs of Theorems 6 and 7 (respectively Theorems 9 and 10), it follows that system (9) (respectively system (8)) with $p(x, y)$ of the form (10) has a weak focus at the origin provided that it has 6 (respectively 2 ) limit cycles up to averaging theory of order 4 .

In the case of limit cycles bifurcating from ovals of the period annulus of a uniform isochronous center, there are examples of quartic polynomial systems which has at least 8 limit cycles, see [13].

All calculations were performed with the assistance of the software Mathematica.

\section{Proof of Theorem 1}

The proof of Theorem 1 is based on the following lemma.

Lemma 11 (Fundamental Lemma). Let $r^{ \pm}(\cdot, \rho, \varepsilon):\left[0, \theta_{\rho}\right) \rightarrow \mathbb{R}^{k}$ be the solution of $r^{\prime}=F^{ \pm}(\theta, r, \varepsilon)$ with $r^{ \pm}(0, \rho, \varepsilon)=\rho$. If $\theta_{\rho}>T$, then

$$
r^{ \pm}(\theta, \rho, \varepsilon)=\rho+\sum_{i=1}^{k} \varepsilon^{i} \frac{y_{i}^{ \pm}(\theta, \rho)}{i !}+\mathcal{O}_{k+1}(\varepsilon),
$$

where $y_{i}^{ \pm}(t, z)$ for $i=1,2, \ldots, k$ are defined in $(3)$.

The proof of Lemma 11 can be found in [16].

Now we prove Theorem 1. First of all we have to show that there exists $\varepsilon_{0}$ sufficietly small such that for each $\rho \in \bar{D}$ and for every $\varepsilon \in\left[-\varepsilon_{0}, \varepsilon_{0}\right]$ the solutions $r^{ \pm}(\theta, \rho, \varepsilon)$ are defined for every $\theta \in[0, T]$. Indeed, by the Existence 
and Uniqueness Theorem of solutions (see, for example, Theorem 1.2.4 of [19]), $r^{ \pm}(\theta, \rho, \varepsilon)$ is defined for all $0 \leq \theta \leq \inf \left(T, d / M^{ \pm}(\varepsilon)\right)$, for each $x$ with $|r-\rho|<d$ and for every $\rho \in \bar{D}$, where

$$
M^{ \pm}(\varepsilon) \geq\left|\sum_{i=1}^{k} \varepsilon^{i} F_{i}^{ \pm}(\theta, \rho)+\varepsilon^{k+1} R^{ \pm}(\theta, \rho, \varepsilon)\right| .
$$

Clearly $\varepsilon$ can be taken sufficiently small in order that inf $\left(T, d / M^{ \pm}(\varepsilon)\right)=T$ for all $\rho \in \bar{D}$. Moreover, since the vector fields $F^{ \pm}(\theta, r, \varepsilon)$ are $T$-periodic, the solutions $r^{ \pm}(\theta, \rho, \varepsilon)$ can be extended for $\theta \in \mathbb{R}$.

We denote

$$
f(\rho, \varepsilon)=r^{+}(\alpha, \rho, \varepsilon)-r^{-}(\alpha-T, \rho, \varepsilon) .
$$

It is easy to see that system (1) for $\varepsilon=\bar{\varepsilon} \in\left(-\varepsilon_{0}, \varepsilon_{0}\right)$ has a periodic solution passing through $\bar{\rho} \in D$ if and only if $f(\bar{\rho}, \bar{\varepsilon})=0$.

From Lemma 11 we have that

$$
\begin{aligned}
f(\rho, \varepsilon) & =\sum_{i=1}^{k} \varepsilon^{i} \frac{y_{i}(\theta, \rho)-y_{i}(\theta, \rho)}{i !}+\mathcal{O}_{k+1}(\varepsilon) \\
& =\sum_{i=1}^{k} \varepsilon^{i} f_{i}(\rho)+\mathcal{O}_{k+1}(\varepsilon)
\end{aligned}
$$

where the function $f_{i}$ is the one defined in (2) for $i=1,2, \cdots, k$. From hypothesis

$$
f(\rho, \varepsilon)=\varepsilon^{r} f_{r}(\rho)+\cdots+\varepsilon^{k} f_{k}(\rho)+\mathcal{O}_{k+1}(\varepsilon) .
$$

Since $f_{r}\left(\rho^{*}\right)=0$ and $f_{r}^{\prime}\left(\rho^{*}\right) \neq 0$, the implicit function theorem applied to the function $\mathcal{F}(\rho, \varepsilon)=f(\rho, \varepsilon) / \varepsilon^{r}$ guarantees the existence of a differentiable function $\rho(\varepsilon)$ such that $\rho(0)=\rho^{*}$ and $f(\rho(\varepsilon), \varepsilon)=\varepsilon^{r} \mathcal{F}(\rho(\varepsilon), \varepsilon)=0$ for every $|\varepsilon| \neq 0$ sufficiently small. Then the proof of the theorem follows.

\section{Proof of Theorem 6}

Consider system (9) with $p(x, y)$ of the general form (10). In order to analyze the Hopf bifurcation for this system, applying Theorem 1 , we set $\alpha=\pi$ and we introduce a small parameter $\varepsilon$ doing the change of coordinates $x=\varepsilon X, y=\varepsilon Y$. After that we perform the polar change of coordinates $X=r \cos \theta, Y=r \sin \theta$, and by doing a Taylor expansion truncated at the $4^{\text {th }}$ order in $\varepsilon$ we obtain an expression for $d r / d \theta$ of the form (1), with $\alpha=\pi$. The explicit expression is quite large so we omit it.

System (9) is a polynomial system, so the functions $F_{i}^{ \pm}(\theta, r)$ and $R_{i}^{ \pm}(\theta, r, \varepsilon)$, $i=1, \ldots, 4$ are analytic, and consequently, locally Lipschitz. Moreover, since the variable $\theta$ appears through sinus and cosinus, system (9) in the form $d r / d \theta$ is 
$2 \pi$-periodic. It suffices to take $D=\left\{r: 0<r<r_{0}\right\}$, where the unperturbed system has periodic solutions passing through the points $(0, r)$ with $0<r<r_{0}$.

We obtain each $y_{i}^{+}$and $y_{i}^{-}, i=1 \ldots, 4$ applying expression (3) respectively for $X_{1}$ and $X_{2}$ of system (9), after the changes described in the first paragraph of this section. Then we calculate the averaged functions $f_{i}, i=1 \ldots, 4$ using equation (2). Hence, by Theorem 1 we have the averaged function of first order

$$
f_{1}(r)=A_{1} r+A_{0},
$$

where

$$
\begin{aligned}
A_{1}= & \frac{1}{2} \pi\left(3 t_{01}\left(\alpha_{0}^{1}+\gamma_{0}^{1}\right)+\alpha_{1}^{1}+\beta_{2}^{1}+\gamma_{1}^{1}+\delta_{2}^{1}-3 t_{10}\left(\beta_{0}^{1}+\delta_{0}^{1}\right)\right), \\
A_{0}= & 2\left(\beta_{2}^{1} \alpha_{0}^{1}+\left(\alpha_{0}^{1}\right)^{2} t_{01}-\beta_{0}^{1}\left(\alpha_{0}^{1} t_{10}+\beta_{1}^{1}\right)-\gamma_{0}^{1} \delta_{2}^{1}-\left(\gamma_{0}^{1}\right)^{2} t_{01}+\delta_{0}^{1}\left(\gamma_{0}^{1} t_{10}+\delta_{1}^{1}\right)\right. \\
& \left.+\beta_{0}^{2}-\delta_{0}^{2}\right) .
\end{aligned}
$$

The rank of the Jacobian matrix of the function $\mathcal{A}=\left(A_{0}, A_{1}\right)$ with respect to the variables $t_{01}, t_{10}, \alpha_{0}^{1}, \alpha_{1}^{1}, \beta_{0}^{1}, \beta_{1}^{1}, \beta_{2}^{1}, \gamma_{0}^{1}, \gamma_{1}^{1}, \delta_{0}^{1}, \delta_{1}^{1}, \delta_{2}^{1}$ is maximal. Then the coefficients $A_{0}$ and $A_{1}$ are linearly independent in their variables.

Clearly $f_{1}(r)$ has at most one solution in $D$. Thus applying Theorem 1 it is proved that at most 1 limit cycle can bifurcate from the origin of system (9) with $p(x, y)$ of the form (10), using the averaging theory of first order. Solving $A_{1}$ for $\alpha_{1}^{1}$ and $A_{0}$ for $\delta_{0}^{2}$ we have $f_{1}(r)=0$, and we can apply the averaging theory of order 2. Its corresponding averaged function is

$$
f_{2}(r)=B_{3} r^{3}+B_{2} r^{2}+B_{1} r+B_{0}
$$

where

$$
\begin{aligned}
B_{3}= & 2 \pi\left(t_{02}+t_{20}\right), \\
B_{2}= & \frac{1}{3}(-4)\left(3 t_{01}\left(2 \alpha_{0}^{1} t_{10}-\alpha_{2}^{1}+4 \gamma_{0}^{1} t_{10}+\gamma_{2}^{1}\right)-8 t_{02}\left(\alpha_{0}^{1}-\gamma_{0}^{1}\right)-\alpha_{0}^{1} t_{20}-\alpha_{4}^{1}-\beta_{3}^{1}\right. \\
& -2 \beta_{5}^{1}+6 \gamma_{1}^{1} t_{10}+\gamma_{0}^{1} t_{20}+\gamma_{4}^{1}+\delta_{3}^{1}+2 \delta_{5}^{1}+3 t_{01}^{2}\left(\beta_{0}^{1}-\delta_{0}^{1}\right)-3 \beta_{0}^{1} t_{10}^{2} \\
& \left.-15 \delta_{0}^{1} t_{10}^{2}+3 \beta_{2}^{1} t_{10}+3 \delta_{2}^{1} t_{10}+4 \beta_{0}^{1} t_{11}-4 \delta_{0}^{1} t_{11}\right), \\
B_{1}= & \frac{1}{4} \pi\left(-8 \alpha_{3}^{1} \beta_{0}^{1}+8 \alpha_{0}^{1} \beta_{5}^{1}-3 t_{01}\left(t _ { 1 0 } \left(-\alpha_{0}^{1} \gamma_{0}^{1}+15 \beta_{0}^{1} \delta_{0}^{1}+8\left(\alpha_{0}^{1}\right)^{2}+8\left(\beta_{0}^{1}\right)^{2}\right.\right.\right. \\
& \left.+\left(\gamma_{0}^{1}\right)^{2}-7\left(\delta_{0}^{1}\right)^{2}\right)+3 \alpha_{2}^{1} \gamma_{0}^{1}-5 \beta_{0}^{1} \delta_{2}^{1}-4 \alpha_{0}^{1} \alpha_{2}^{1}-4 \beta_{0}^{1} \beta_{2}^{1}-5 \beta_{0}^{1} \gamma_{1}^{1} \\
& \left.-\beta_{1}^{1} \gamma_{0}^{1}+5 \gamma_{1}^{1} \delta_{0}^{1}+\gamma_{0}^{1} \delta_{1}^{1}-7 \gamma_{0}^{1} \gamma_{2}^{1}+\delta_{0}^{1} \delta_{2}^{1}-4 \alpha_{0}^{2}-4 \gamma_{0}^{2}\right) \\
& +3 t_{01}^{2}\left(8 \alpha_{0}^{1} \beta_{0}^{1}+\gamma_{0}^{1}\left(15 \beta_{0}^{1}-7 \delta_{0}^{1}\right)\right)+16 t_{02}\left(\left(\alpha_{0}^{1}\right)^{2}+\left(\gamma_{0}^{1}\right)^{2}\right)+\alpha_{0}^{1} \gamma_{1}^{1} t_{10} \\
& -3 \alpha_{2}^{1} \gamma_{1}^{1}-3 \alpha_{2}^{1} \delta_{2}^{1}+\beta_{1}^{1} \delta_{2}^{1}+24 \alpha_{0}^{1} \beta_{0}^{1} t_{10}^{2}-3 \alpha_{0}^{1} \delta_{0}^{1} t_{10}^{2} \\
& -24 \alpha_{0}^{1} \beta_{2}^{1} t_{10}+9 \alpha_{2}^{1} \delta_{0}^{1} t_{10}+\alpha_{0}^{1} \delta_{2}^{1} t_{10}-16 \alpha_{0}^{1} \beta_{0}^{1} t_{11}+4 \alpha_{0}^{1} \alpha_{4}^{1}-4 \beta_{0}^{1} \beta_{4}^{1} \\
& +\beta_{1}^{1} \gamma_{1}^{1}-8 \gamma_{3}^{1} \delta_{0}^{1}-\gamma_{1}^{1} \delta_{1}^{1}+3 \gamma_{2}^{1} \delta_{2}^{1}+8 \gamma_{0}^{1} \delta_{5}^{1}+3 \gamma_{0}^{1} \delta_{0}^{1} t_{10}^{2}
\end{aligned}
$$




$$
\begin{aligned}
& -9 \gamma_{2}^{1} \delta_{0}^{1} t_{10}-\gamma_{0}^{1} \delta_{2}^{1} t_{10}-\gamma_{0}^{1} \gamma_{1}^{1} t_{10}-16 \gamma_{0}^{1} \delta_{0}^{1} t_{11}+3 \gamma_{1}^{1} \gamma_{2}^{1}+4 \gamma_{0}^{1} \gamma_{4}^{1} \\
& -\delta_{1}^{1} \delta_{2}^{1}-4 \delta_{0}^{1} \delta_{4}^{1}+4 \alpha_{1}^{2}+4 \beta_{2}^{2}+4 \gamma_{1}^{2}+4 \delta_{2}^{2}-3 \beta_{1}^{1} \delta_{0}^{1} t_{10} \\
& \left.+24 \beta_{0}^{1} \beta_{1}^{1} t_{10}+3 \delta_{0}^{1} \delta_{1}^{1} t_{10}-24 \beta_{0}^{2} t_{10}+16\left(\beta_{0}^{1}\right)^{2} t_{20}+16\left(\delta_{0}^{1}\right)^{2} t_{20}\right), \\
& B_{0}=-4\left(-\alpha_{0}^{1} \beta_{2}^{1} \delta_{1}^{1}+\alpha_{0}^{1} \beta_{1}^{1} \beta_{2}^{1}+\alpha_{0}^{1} \beta_{0}^{1} \beta_{4}^{1}-\left(\alpha_{0}^{1}\right)^{2} \beta_{5}^{1}+t_{01}\left(\left(\alpha_{0}^{1}\right)^{2}\left(\beta_{1}^{1}-\delta_{1}^{1}\right)\right.\right. \\
& +\alpha_{0}^{1}\left(3 \alpha_{2}^{1} \gamma_{0}^{1}+2 \beta_{0}^{1}\left(\gamma_{1}^{1}+\delta_{2}^{1}\right)\right)+t_{10}\left(-6 \beta_{0}^{1} \alpha_{0}^{1} \delta_{0}^{1}-\left(\alpha_{0}^{1}\right)^{2} \gamma_{0}^{1}+\left(\alpha_{0}^{1}\right)^{3}\right. \\
& \left.-6 \gamma_{0}^{1}\left(3 \beta_{0}^{1} \delta_{0}^{1}+\left(\beta_{0}^{1}\right)^{2}-\left(\delta_{0}^{1}\right)^{2}\right)\right)+\gamma_{0}^{1}\left(6 \beta_{0}^{1} \delta_{2}^{1}+3 \beta_{0}^{1} \beta_{2}^{1}+6 \beta_{0}^{1} \gamma_{1}^{1}\right. \\
& \left.\left.-4 \gamma_{1}^{1} \delta_{0}^{1}+3 \gamma_{0}^{1} \gamma_{2}^{1}-\delta_{0}^{1} \delta_{2}^{1}+3 \alpha_{0}^{2}+3 \gamma_{0}^{2}\right)\right)+3 \gamma_{0}^{1} t_{01}^{2}\left(2 \alpha_{0}^{1} \beta_{0}^{1}\right. \\
& \left.+\gamma_{0}^{1}\left(3 \beta_{0}^{1}-\delta_{0}^{1}\right)\right)+t_{02}\left(\left(\gamma_{0}^{1}\right)^{3}-\left(\alpha_{0}^{1}\right)^{3}\right)+\alpha_{0}^{1} \beta_{0}^{1} \gamma_{0}^{1} t_{10}^{2}-\alpha_{0}^{1} \beta_{2}^{1} \gamma_{0}^{1} t_{10} \\
& +\beta_{0}^{1}\left(\delta_{2}^{1}\right)^{2}+\alpha_{0}^{1} \alpha_{2}^{1} \gamma_{1}^{1}+\beta_{0}^{1} \beta_{1}^{1} \delta_{1}^{1}+\alpha_{0}^{1} \alpha_{2}^{1} \delta_{2}^{1}+\beta_{0}^{1} \beta_{2}^{1} \delta_{2}^{1} \\
& +\beta_{1}^{1} \beta_{0}^{2}+\beta_{0}^{1} \beta_{1}^{2}-\alpha_{0}^{1} \beta_{2}^{2}-\left(\alpha_{0}^{1}\right)^{2} \beta_{0}^{1} t_{10}^{2}+\alpha_{0}^{1} \beta_{0}^{1} \delta_{1}^{1} t_{10}-2 \alpha_{0}^{1} \beta_{0}^{1} \beta_{1}^{1} t_{10} \\
& +\left(\alpha_{0}^{1}\right)^{2} \beta_{2}^{1} t_{10}-3 \alpha_{0}^{1} \alpha_{2}^{1} \delta_{0}^{1} t_{10}+\alpha_{0}^{1} \beta_{0}^{2} t_{10}+\left(\alpha_{0}^{1}\right)^{2} \beta_{0}^{1} t_{11}-\alpha_{0}^{1}\left(\beta_{0}^{1}\right)^{2} t_{20} \\
& -\beta_{0}^{1}\left(\beta_{1}^{1}\right)^{2}-\left(\beta_{0}^{1}\right)^{2} \beta_{3}^{1}+2 \beta_{0}^{1} \gamma_{1}^{1} \delta_{2}^{1}+\beta_{0}^{1}\left(\gamma_{1}^{1}\right)^{2}+\beta_{0}^{1} \beta_{2}^{1} \gamma_{1}^{1}-\left(\gamma_{1}^{1}\right)^{2} \delta_{0}^{1} \\
& -\gamma_{1}^{1} \delta_{2}^{1} \delta_{0}^{1}-\gamma_{0}^{1} \delta_{4}^{1} \delta_{0}^{1}+\gamma_{0}^{1} \gamma_{2}^{1} \delta_{2}^{1}+\left(\gamma_{0}^{1}\right)^{2} \delta_{5}^{1}+\gamma_{1}^{1} \alpha_{0}^{2}-\delta_{1}^{1} \beta_{0}^{2} \\
& -\delta_{0}^{1} \delta_{1}^{2}+\gamma_{1}^{1} \gamma_{0}^{2}+\gamma_{0}^{1} \delta_{2}^{2}-6 \beta_{0}^{1} \gamma_{1}^{1} \delta_{0}^{1} t_{10}-2\left(\beta_{0}^{1}\right)^{2} \gamma_{1}^{1} t_{10} \\
& +\beta_{0}^{1} \beta_{1}^{1} \gamma_{0}^{1} t_{10}+4 \gamma_{1}^{1}\left(\delta_{0}^{1}\right)^{2} t_{10}-3 \gamma_{0}^{1} \gamma_{2}^{1} \delta_{0}^{1} t_{10}-\gamma_{0}^{1} \beta_{0}^{2} t_{10}-\left(\gamma_{0}^{1}\right)^{2} \delta_{0}^{1} t_{11} \\
& +\gamma_{0}^{1}\left(\delta_{0}^{1}\right)^{2} t_{20}+\delta_{3}^{1}\left(\delta_{0}^{1}\right)^{2}+\gamma_{0}^{1} \gamma_{1}^{1} \gamma_{2}^{1}+\delta_{2}^{1} \alpha_{0}^{2}-3 \delta_{0}^{1} \alpha_{0}^{2} t_{10} \\
& +\delta_{2}^{1} \gamma_{0}^{2}-3 \delta_{0}^{1} \gamma_{0}^{2} t_{10}-\beta_{0}^{3}+\delta_{0}^{3}+9 \beta_{0}^{1}\left(\delta_{0}^{1}\right)^{2} t_{10}^{2}+6\left(\beta_{0}^{1}\right)^{2} \delta_{0}^{1} t_{10}^{2} \\
& \left.-3\left(\delta_{0}^{1}\right)^{3} t_{10}^{2}-3 \beta_{0}^{1} \beta_{2}^{1} \delta_{0}^{1} t_{10}-6 \beta_{0}^{1} \delta_{2}^{1} \delta_{0}^{1} t_{10}-2\left(\beta_{0}^{1}\right)^{2} \delta_{2}^{1} t_{10}+\delta_{2}^{1}\left(\delta_{0}^{1}\right)^{2} t_{10}\right) \text {, }
\end{aligned}
$$

and since the rank of the Jacobian matrix of the function $\mathcal{B}=\left(B_{0}, B_{1}, B_{2}, B_{3}\right)$ with respect to its variables is maximal, $B_{i}, i=0, \ldots, 3$ are linearly independent in their variables.

Hence $f_{2}(r)$ has at most 3 solutions in $D$, see Theorem 5. Applying Theorem 1 it is proved that at most 3 limit cycles can bifurcate from the origin of system (9) with $p(x, y)$ of the form (10), using the averaging theory of order 2. Solving $B_{3}$ for $t_{02}, B_{2}$ for $\alpha_{4}^{1}, B_{1}$ for $\beta_{2}^{2}$ and $B_{0}$ for $\delta_{0}^{3}$ we obtain $f_{2}(r)=0$, and we can apply the averaging theory of order 3 , which corresponding averaged function is of the form

$$
r f_{3}(r)=C_{4} r^{4}+C_{3} r^{3}+C_{2} r^{2}+C_{1} r+C_{0}
$$

and $C_{i}$ for $i=0, \ldots, 4$ are linearly independent in their variables, because the rank of the Jacobian matrix of the function $\mathcal{C}=\left(C_{0}, \ldots, C_{4}\right)$ with respect to its variables is maximal. We do not explicitly provide their expressions, since they are very long. Therefore $f_{3}(r)$ has at most 4 solutions in $D$, by Theorem 5. Applying Theorem 1 it is proved that at most 4 limit cycles can bifurcate from the origin of system (9) with $p(x, y)$ of the form (10) using the averaging 
theory of order 3. By conveniently choosing variables to cancel the coefficients $C_{i}, i=0, \ldots, 4$ we have $f_{3}(r)=0$. Hence we apply the averaging theory of order 4 to obtain the averaged function of order 4

$$
r f_{4}(r)=D_{6} r^{6}+D_{5} r^{5}+D_{4} r^{4}+D_{3} r^{3}+D_{2} r^{2}+D_{1} r+D_{0} .
$$

Since the rank of the Jacobian matrix of the function $\mathcal{D}=\left(D_{0}, \ldots, D_{6}\right)$ with respect to its variables is maximal, the coefficients $D_{i}, i=0, \ldots, 6$ are linearly independent in their variables. Their expressions are very long so we do not provide them here. As a result of these calculations, it follows that $f_{4}(r)$ has at most 6 solutions in $D$ by Theorem 5 . Applying Theorem 1 we conclude that at most 6 limit cycles can bifurcate from the origin of system (9) with $p(x, y)$ of the form (10), using the averaging theory of order 4 . This result is a lower bound for $H_{d}(4)$, hence Theorem 6 is proved.

\section{Proof of Theorem 7}

First we consider the systems of the form (9) with $p(x, y)$ of the form (11). According to Theorem 3, the corresponding unperturbed system has a uniform isochronous center at the origin. In order to study the Hopf bifurcation for this case, we apply the results obtained in the proof of Theorem 6 , by conveniently vanishing the coefficients of (10), used in that proof. More precisely, we take $t_{01}=t_{20}=t_{02}=t_{21}=t_{03}=0$.

We also consider the systems of the form (9), with $p(x, y)$ of the form (12), whose corresponding unperturbed system also has a uniform isochronous center at the origin, see Theorem 4. Again, we use the results obtained in the proof of Theorem 6, vanishing the appropriate coefficients of (10), that is, we take $t_{01}=t_{10}=t_{20}=t_{11}=t_{02}=0$.

Considering the above restrictions to the coefficients of $p(x, y)$ we obtain the averaged functions $f_{i}, i=1, \ldots, 4$ and since they are similar to those calculated in the proof of Theorem 6 we do not explicitly present them here. It is interesting to observe that the same number of limit cycles in each averaging order was obtained with $p(x, y)$ of the form (11) and (12).

The following table summarizes the results obtained in this proof and in the proof of Theorem 6 .

It follows that if system (9) has 6 limit cycles up to the averaging theory of order 4 , then it must have a weak focus at the origin.

\section{Proof of Theorem 8}

Consider system (9) with $p(x, y)$ of the form (11) and take $\alpha_{0}^{j}=\beta_{0}^{j}=\gamma_{0}^{j}=$ $\delta_{0}^{j}=0$, for $j=1, \ldots, 7$. In this case the corresponding unperturbed system has a uniform isochronous center at the origin, see Theorem 3. In order to analyze the Hopf bifurcation for this case, applying Theorem 1, we set $\alpha=\pi$ and we 


\begin{tabular}{|c|c|c|}
\hline \multirow{2}{*}{ Averaging order } & \multicolumn{2}{|c|}{ \# limit cycles } \\
\cline { 2 - 3 } & Theorem 6 & Theorem 7 with $p(x, y)$ given by $(11)$ or $(12)$ \\
\hline \hline 1 & 1 & 1 \\
\hline 2 & 3 & 2 \\
\hline 3 & 4 & 4 \\
\hline 4 & 6 & 5 \\
\hline
\end{tabular}

TABLE 1. Number of limit cycles for discontinuous differential systems (9).

introduce a small parameter $\varepsilon$ doing the rescaling $x=\varepsilon X, y=\varepsilon Y$. After that doing the polar change of coordinates $X=r \cos \theta, Y=r \sin \theta$ and a Taylor expansion truncated at the $7^{\text {th }}$ order in $\varepsilon$ we obtain an expression for $d r / d \theta$ of the form (1), with $\alpha=\pi$. The explicit expression is quite large so we omit it. All hypotheses for applying Theorem 1 to this case are satisfied using similar arguments to those presented for the proof of Theorem 6 .

We obtain each $y_{i}^{+}$and $y_{i}^{-}, i=1 \ldots, 7$ applying expression (3) respectively for $X_{1}$ and $X_{2}$ of system (9), after the changes previously described. Then we calculate the averaged functions $f_{i}, i=1 \ldots, 7$ using equation (2). We remark that, up to the averaging theory of order 4 , the results in this case can be easily obtained from those already calculated in the proof of Theorem 7 , taking into account the condition $\alpha_{0}^{j}=\beta_{0}^{j}=\gamma_{0}^{j}=\delta_{0}^{j}=0, j=1, \ldots, 7$, so we do not explicitly present the averaging functions from order 1 to 3 here. Starting from the averaged function of order 4 we have

$$
f_{4}(r)=R_{4} r^{4}+R_{3} r^{3}+R_{2} r^{2}+R_{1} r,
$$

and $R_{i}$ for $i=1, \ldots, 4$ are linearly independent in their variables, since the rank of the Jacobian matrix of the function $\mathcal{R}=\left(R_{1}, \ldots, R_{4}\right)$ with respect to its variables is maximal. We do not explicitly provide their expressions, because they are very long. Therefore $f_{4}(r)$ has at most 3 solutions in $D$, by Theorem 5 . Applying Theorem 1 it is proved that at most 3 limit cycles can bifurcate from the origin of system (9) with $p(x, y)$ of the form (11), and $\alpha_{0}^{j}=\beta_{0}^{j}=\gamma_{0}^{j}=\delta_{0}^{j}=0, j=1, \ldots, 7$ using the averaging theory of order 4 .

The next averaging functions are calculated in a similar way, so we obtain

$$
f_{5}(r)=S_{5} r^{5}+S_{4} r^{4}+S_{3} r^{3}+S_{2} r^{2}+S_{1} r
$$

and $S_{i}$ for $i=1, \ldots, 5$ are linearly independent in their variables,

$$
f_{6}(r)=T_{6} r^{6}+T_{5} r^{5}+T_{4} r^{4}+T_{3} r^{3}+T_{2} r^{2}+T_{1} r,
$$

and $T_{j}$ for $j=1, \ldots, 6$ are linearly independent in their variables,

$$
f_{7}(r)=U_{7} r^{7}+U_{6} r^{6}+U_{5} r^{5}+U_{4} r^{4}+U_{3} r^{3}+U_{2} r^{2}+U_{1} r
$$


and $U_{k}$ for $k=1, \ldots, 7$ are linearly independent in their variables. The expressions of $S_{i}, i=1, \ldots, 5, T_{j}, j=1, \ldots, 6$ and $U_{k}, k=1, \ldots, 7$ are very long so we do not provide them here.

Thus $f_{5}(r), f_{6}(r)$ and $f_{7}(r)$ has at most 4,5 and 6 solutions in $D$, respectively, see Theorem 5. Applying Theorem 1 we conclude that at most 4, 5, and 6 limit cycles can bifurcate from the origin of system (9) with $p(x, y)$ of the form (11), and $\alpha_{0}^{j}=\beta_{0}^{j}=\gamma_{0}^{j}=\delta_{0}^{j}=0, j=1, \ldots, 7$ using the averaging theory of order 5,6 and 7 , respectively. Therefore Theorem 8 is proved.

The following table summarizes our results for this case

\begin{tabular}{|c|c|}
\hline Averaging order & \# limit cycles \\
\hline \hline 1 & 0 \\
\hline 2 & 1 \\
\hline 3 & 2 \\
\hline 4 & 3 \\
\hline 5 & 4 \\
\hline 6 & 5 \\
\hline 7 & 6 \\
\hline
\end{tabular}

TABLE 2. Limit cycles for quartic discontinuous differential systems with a uniform isochronous center at the origin. 


\section{Proof of Theorems 9 And 10}

System (1) becomes continuous by taking $\alpha=2 \pi$ and therefore the averaging theory developed in subsection 1.1 also applies to continuous differential systems.

First, consider the continuous differential system (8) with $p(x, y)$ of the form (10). In order to study the limit cycles for this system we only need the expressions of $y_{i}^{+}, i=1 \ldots, 4$, which were already calculated for studying the previous cases. Hence, the averaged functions $f_{i}, i=1 \ldots, 4$ can be obtained by the same algorithm used for the discontinuous differential systems, by taking $\alpha=2 \pi$.

The unperturbed continuous differential system corresponding to the perturbed system (8), with either $p(x, y)$ of the form (11) or (12) has a uniform isochronous center at the origin, according to Theorems 3 and 4, respectively. We apply the same arguments as in the previous paragraph, by taking $\alpha=2 \pi$ and using the expressions of $y_{i}^{+}, i=1 \ldots, 4$ calculated in the proof of Theorem 7 to obtain the averaged functions $f_{i}, i=1 \ldots, 4$ for this case. We remark that the same number of limit cycles was obtained in both cases where $p(x, y)$ is either of the form (11) or (12), in each averaging order studied.

Since the calculations and arguments are quite similar to those used in the previous proofs, we omit the explicit expressions of the averaged functions. We summarize our results in the following table

\begin{tabular}{l|l|c|c|}
\hline \multirow{2}{*}{ Averaging order } & \multicolumn{2}{|c|}{ \# limit cycles } \\
\cline { 2 - 4 } & general case & Uniform center \\
\hline \hline 1 & 0 & 0 \\
\hline 2 & 1 & 0 \\
\hline 3 & 1 & 1 \\
\hline 4 & 2 & 1 \\
\hline TABLE & 3. Number of limit cycles for continuous differential \\
systems (8).
\end{tabular}

We remark that from this proof, it follows that system (8) with $p(x, y)$ of the form (10) has a weak focus at the origin provided that it has 2 limit cycles up to the averaging theory of order 4 .

\section{Appendix}

$$
\begin{aligned}
& y_{1}^{ \pm}(\theta, \rho)=\int_{0}^{\theta} F_{1}^{ \pm}(\phi, \rho) d \phi \\
& y_{2}^{ \pm}(\theta, \rho)=\int_{0}^{\theta}\left(2 F_{2}^{ \pm}(\phi, \rho)+2 \partial F_{1}^{ \pm}(\phi, \rho) y_{1}^{ \pm}(\phi, \rho)\right) d \phi
\end{aligned}
$$




$$
\begin{aligned}
& y_{3}^{ \pm}(\theta, \rho)=\int_{0}^{\theta}\left(6 F_{3}^{ \pm}(\phi, \rho)+6 \partial F_{2}^{ \pm}(\phi, \rho) y_{1}^{ \pm}(\phi, \rho)\right. \\
& \left.+3 \partial^{2} F_{1}^{ \pm}(\phi, \rho) y_{1}^{ \pm}(\phi, \rho)^{2}+3 \partial F_{1}^{ \pm}(\phi, \rho) y_{2}^{ \pm}(\phi, \rho)\right) d \phi, \\
& y_{4}^{ \pm}(\theta, \rho)=\int_{0}^{\theta}\left(24 F_{4}^{ \pm}(\phi, \rho)+24 \partial F_{3}^{ \pm}(\phi, \rho) y_{1}^{ \pm}(\phi, \rho)\right. \\
& +12 \partial^{2} F_{2}^{ \pm}(\phi, \rho) y_{1}^{ \pm}(\phi, \rho)^{2}+12 \partial F_{2}^{ \pm}(\phi, \rho) y_{2}^{ \pm}(\phi, \rho) \\
& +12 \partial^{2} F_{1}^{ \pm}(\phi, \rho) y_{1}^{ \pm}(\phi, \rho) y_{2}^{ \pm}(\phi, \rho) \\
& \left.+4 \partial^{3} F_{1}^{ \pm}(\phi, \rho) y_{1}^{ \pm}(\phi, \rho)^{3}+4 \partial F_{1}^{ \pm}(\phi, \rho) y_{3}^{ \pm}(\phi, \rho)\right) d \phi, \\
& y_{5}^{ \pm}(\theta, \rho)=\int_{0}^{\theta}\left(120 F_{5}^{ \pm}(\phi, \rho)+120 \partial F_{4}^{ \pm}(\phi, \rho) y_{1}^{ \pm}(\phi, \rho)\right. \\
& +60 \partial^{2} F_{3}^{ \pm}(\phi, \rho) y_{1}^{ \pm}(\phi, \rho)^{2}+60 \partial F_{3}^{ \pm}(\phi, \rho) y_{2}^{ \pm}(\phi, \rho) \\
& +60 \partial^{2} F_{2}^{ \pm}(\phi, \rho) y_{1}^{ \pm}(\phi, \rho) y_{2}^{ \pm}(\phi, \rho)+20 \partial^{3} F_{2}^{ \pm}(\phi, \rho) y_{1}^{ \pm}(\phi, \rho)^{3} \\
& +20 \partial F_{2}^{ \pm}(\phi, \rho) y_{3}^{ \pm}(\phi, \rho)+20 \partial^{2} F_{1}^{ \pm}(\phi, \rho) y_{1}^{ \pm}(\phi, \rho) y_{3}^{ \pm}(\phi, \rho) \\
& +15 \partial^{2} F_{1}^{ \pm}(\phi, \rho) y_{2}^{ \pm}(\phi, \rho)^{2}+30 \partial^{3} F_{1}^{ \pm}(\phi, \rho) y_{1}^{ \pm}(\phi, \rho)^{2} y_{2}^{ \pm}(\phi, \rho) \\
& \left.+5 \partial^{4} F_{1}^{ \pm}(\phi, \rho) y_{1}^{ \pm}(\phi, \rho)^{4}+5 \partial F_{1}^{ \pm}(\phi, \rho) y_{4}^{ \pm}(\phi, \rho)\right) d \phi, \\
& y_{6}^{ \pm}(\theta, \rho)=\int_{0}^{\theta}\left(720 F_{6}^{ \pm}(\phi, \rho)+720 \partial F_{5}^{ \pm}(\phi, \rho) y_{1}^{ \pm}(\phi, \rho)\right. \\
& +360 \partial^{2} F_{4}^{ \pm}(\phi, \rho) y_{1}^{ \pm}(\phi, \rho)^{2}+360 \partial F_{4}^{ \pm}(\phi, \rho) y_{2}^{ \pm}(\phi, \rho) \\
& +120 \partial^{3} F_{3}^{ \pm}(\phi, \rho) y_{1}^{ \pm}(\phi, \rho)^{3}+360 \partial^{2} F_{3}^{ \pm}(\phi, \rho) y_{1}^{ \pm}(\phi, \rho) y_{2}^{ \pm}(\phi, \rho) \\
& +120 \partial F_{3}^{ \pm}(\phi, \rho) y_{3}^{ \pm}(\phi, \rho)+30 \partial^{4} F_{2}^{ \pm}(\phi, \rho) y_{1}^{ \pm}(\phi, \rho)^{4} \\
& +180 \partial^{3} F_{2}^{ \pm}(\phi, \rho) y_{1}^{ \pm}(\phi, \rho)^{2} y_{2}^{ \pm}(\phi, \rho)+120 \partial^{2} F_{2}^{ \pm}(\phi, \rho) y_{1}^{ \pm}(\phi, \rho) y_{3}^{ \pm}(\phi, \rho) \\
& +90 \partial^{2} F_{2}^{ \pm}(\phi, \rho) y_{2}^{ \pm}(\phi, \rho)^{2}+30 \partial F_{2}^{ \pm}(\phi, \rho) y_{4}^{ \pm}(\phi, \rho) \\
& +60 \partial^{4} F_{1}^{ \pm}(\phi, \rho) y_{1}^{ \pm}(\phi, \rho)^{3} y_{2}^{ \pm}(\phi, \rho)+60 \partial^{3} F_{1}^{ \pm}(\phi, \rho) y_{1}^{ \pm}(\phi, \rho)^{2} y_{3}^{ \pm}(\phi, \rho) \\
& +90 \partial^{3} F_{1}^{ \pm}(\phi, \rho) y_{1}^{ \pm}(\phi, \rho) y_{2}^{ \pm}(\phi, \rho)^{2}+30 \partial^{2} F_{1}^{ \pm}(\phi, \rho) y_{1}^{ \pm}(\phi, \rho) y_{4}^{ \pm}(\phi, \rho) \\
& +60 \partial^{2} F_{1}^{ \pm}(\phi, \rho) y_{2}^{ \pm}(\phi, \rho) y_{3}^{ \pm}(\phi, \rho)+6 \partial^{5} F_{1}^{ \pm}(\phi, \rho) y_{1}^{ \pm}(\phi, \rho)^{5} \\
& \left.+6 \partial F_{1}^{ \pm}(\phi, \rho) y_{5}^{ \pm}(\phi, \rho)\right) d \phi, \\
& y_{7}^{ \pm}(t, \rho)=\int_{0}^{t}\left(5040 F_{7}^{ \pm}(\phi, \rho)+5040 \partial F_{6}^{ \pm}(\phi, \rho) y_{1}^{ \pm}(\phi, \rho)\right. \\
& +2520 \partial^{2} F_{5}^{ \pm}(\phi, \rho) y_{1}^{ \pm}(\phi, \rho)^{2}+2520 \partial F_{5}^{ \pm}(\phi, \rho) y_{2}^{ \pm}(\phi, \rho) \\
& +2520 \partial^{2} F_{4}^{ \pm}(\phi, \rho) y_{1}^{ \pm}(\phi, \rho) y_{2}^{ \pm}(\phi, \rho)+840 \partial^{3} F_{4}^{ \pm}(\phi, \rho) y_{1}^{ \pm}(\phi, \rho)^{3}
\end{aligned}
$$




$$
\begin{aligned}
& +840 \partial F_{4}^{ \pm}(\phi, \rho) y_{3}^{ \pm}(\phi, \rho)+840 \partial^{2} F_{3}^{ \pm}(\phi, \rho) y_{1}^{ \pm}(\phi, \rho) y_{3}^{ \pm}(\phi, \rho) \\
& +630 \partial^{2} F_{3}^{ \pm}(\phi, \rho) y_{2}^{ \pm}(\phi, \rho)^{2}+1260 \partial^{3} F_{3}^{ \pm}(\phi, \rho) y_{1}^{ \pm}(\phi, \rho)^{2} y_{2}^{ \pm}(\phi, \rho) \\
& +210 \partial^{4} F_{3}^{ \pm}(\phi, \rho) y_{1}^{ \pm}(\phi, \rho)^{4}+210 \partial F_{3}^{ \pm}(\phi, \rho) y_{4}^{ \pm}(\phi, \rho) \\
& +210 \partial^{2} F_{2}^{ \pm}(\phi, \rho) y_{1}^{ \pm}(\phi, \rho) y_{4}^{ \pm}(\phi, \rho)+420 \partial^{3} F_{2}^{ \pm}(\phi, \rho) y_{1}^{ \pm}(\phi, \rho)^{2} y_{3}^{ \pm}(\phi, \rho) \\
& +420 \partial^{4} F_{2}^{ \pm}(\phi, \rho) y_{1}^{ \pm}(\phi, \rho)^{3} y_{2}^{ \pm}(\phi, \rho)+630 \partial^{3} F_{2}^{ \pm}(\phi, \rho) y_{2}^{ \pm}(\phi, \rho)^{2} y_{1}^{ \pm}(\phi, \rho) \\
& +42 \partial^{5} F_{2}^{ \pm}(\phi, \rho) y_{1}^{ \pm}(\phi, \rho)^{5}+420 \partial^{2} F_{2}^{ \pm}(\phi, \rho) y_{2}^{ \pm}(\phi, \rho) y_{3}^{ \pm}(\phi, \rho) \\
& +42 \partial F_{2}^{ \pm}(\phi, \rho) y_{5}^{ \pm}(\phi, \rho)+630 \partial^{3} F_{2}^{ \pm}(\phi, \rho) y_{2}^{ \pm}(\phi, \rho)^{2} y_{1}^{ \pm}(\phi, \rho) \\
& +7 \partial^{6} F_{1}^{ \pm}(\phi, \rho) y_{1}^{ \pm}(\phi, \rho)^{6}+105 \partial^{5} F_{1}^{ \pm}(\phi, \rho) y_{1}^{ \pm}(\phi, \rho)^{4} y_{2}^{ \pm}(\phi, \rho) \\
& +140 \partial^{4} F_{1}^{ \pm}(\phi, \rho) y_{1}^{ \pm}(\phi, \rho)^{3} y_{3}^{ \pm}(\phi, \rho)+630 \partial^{4} F_{1}^{ \pm}(\phi, \rho) y_{1}^{ \pm}(\phi, \rho)^{2} y_{2}^{ \pm}(\phi, \rho)^{2} \\
& +105 \partial^{3} F_{1}^{ \pm}(\phi, \rho) y_{1}^{ \pm}(\phi, \rho)^{2} y_{4}^{ \pm}(\phi, \rho)+42 \partial^{2} F_{1}^{ \pm}(\phi, \rho) y_{1}^{ \pm}(\phi, \rho) y_{5}^{ \pm}(\phi, \rho) \\
& +420 \partial^{3} F_{1}^{ \pm}(\phi, \rho) y_{1}^{ \pm}(\phi, \rho) y_{2}^{ \pm}(\phi, \rho) y_{3}^{ \pm}(\phi, \rho) \\
& +105 \partial^{3} F_{1}^{ \pm}(\phi, \rho) y_{2}^{ \pm}(\phi, \rho)^{3}+105 \partial^{2} F_{1}^{ \pm}(\phi, \rho) y_{2}^{ \pm}(\phi, \rho) y_{4}^{ \pm}(\phi, \rho) \\
& \left.+70 \partial^{2} F_{1}^{ \pm}(\phi, \rho) y_{3}^{ \pm}(\phi, \rho)^{2}+7 \partial F_{1}^{ \pm}(\phi, \rho) y_{6}^{ \pm}(\phi, \rho)\right) d \phi .
\end{aligned}
$$

\section{ACKNOWLEDGEMENTS}

The first author is supported by a Ciência sem Fronteiras-CNPq grant number 201002/ 2012-4. The second author is partially supported by a MINECO/FEDER grant number MTM2009-03437, by an AGAUR grant number 2014SGR-568, by an ICREA Academia, two FP7+PEOPLE+2012+IRS8S numbers 316338 and 318999, and UNAB13-4E-1604. The third author is partially supported by a FAPESP-BRAZIL grant 2012/10231-7. The second and third authors are also supported by a CAPES CSF-PVE grant 88881.030454/ 2013-01 from the program CSF-PVE.

\section{REFERENCES}

[1] A. Algaba And M. Reyes, Computing center conditions for vector fields with constant angular speed, J. Comp. and Appl. Math. 154 (2003), 143-159.

[2] A. Algaba, M. Reyes, T. Ortega and A. Bravo, Campos curticos con velocidad angular constante, Actas : XVI CEDYA Congreso de Ecuaciones Diferenciales y Aplicaciones, VI CMA Congreso de Matemtica Aplicada, Las Palmas de Gran Canaria 2 (1999), 1341-1348.

[3] M.A.M. Alwash AND N.G. LlOyd, Non-autonomous equations related to polynomial two-dimensions systems, Proc. Roy. Soc. Eddinburgh Sect. A 105 (1987), 129-152.

[4] I.S. Berezin AND N.P. Zhidkov, Computing Methods, Volume II, Pergamon Press, Oxford, 1964.

[5] M. di Bernardo; C.J. Budd, A. R. Champneys and P. Kowalczyk, Piecewisesmooth dynamical systems: theory and applications, Appl. Math. Sci. 163, Spring-Verlag, London, 2008. 
[6] J. Chavarriga and M. Sabatini, A survey of isochronous centers, Qualitative Theory of Dynamical Systems 1 (1999), 1-70.

[7] J. Chavarriga, I.A. García, J. Giné, On the integrability of differential equations defined by the sum of homogeneous vector fields with degenerate infinity, Internat. J. Bifur. Chaos Appl. Sci. Engrg. 11 (2001), 711-722.

[8] R. Conti, Uniformly isochronous centers of polynomial systems in $\mathbb{R}^{2}$, Lecture Notes in Pure and Appl. Math. 152 (1994), 21-31.

[9] M. Han And V.G. Romanovski, Isochronicity and normal forms of polynomial systems of ODEs, J. Symb. Comput. 47 (2012), 1163-1174.

[10] D. Hilbert, Mathematische probleme (lecture), in: Second Internat. Congress Math., Paris, 1900, in: Nach. Ges. Wiss. Gottingen Math.-Phys. Kl., 1900, 253-297.

[11] J. ITIKAWA AND J. LliBRe, Limit cycles for continuous and discontinuous perturbations of uniform isochronous cubic centers, J. Comp. and Appl. Math. 277 (2014), 171-191.

[12] J. ItIKAWA AND J. LliBRe, Phase portraits of uniform isochronous quartic centers, to appear in J. Comp. and Appl. Math.

[13] J. ITIKAWA AND J. LLIBRE, Limit cycles bifurcanting from a quartic polynomial differential system, Preprint, 2014.

[14] J. Llibre, A.C. Mereu And D.D. Novaes, Averaging theory for discontinuous piecewise differential systems, to appear in J. Diff. Equations.

[15] J. LliBRE AND D.D. NOvAES, On the periodic solutions of discontinuous piecewise differential systems, Preprint, 2014.

[16] J. Llibre, D.D. Novaes And M.A. Teixeira, Higher order averaging theory for finding periodic solutions via Brouwer degree, Nonlinearity 27 (2014), 563-583.

[17] J. Llibre, D.D. Novaes And M.A. TeixeIRA, On the birth of limit cycles for non-smooth dynamical systems, Bulletin des Sciences Mathemtiques, DOI: 10.1016/j.bulsci.2014.08.011 (2014).

[18] A.E. Rudenok, Strong isochronism of a center. Periods of limit cycles of Lienard's system, Differential Equations 11 (1975), 610-617.

[19] J.A. Sanders, F. Verhulst And J. Murdock, Averaging methods in nonlinear dynamical systems, 2nd, edition, Applied Mathematical Sciences 59, Springer, New York, 2007.

[20] Y. Zou, T. Kupper And W.J. Beyn, Generalized Hopf bifurcation for planar Filippov systems continuous at the origin, J. Nonlinear Science 16 (2006), 159-177.

${ }^{1}$ Departament de Matemàtiques, Universitat Autònoma de Barcelona, 08193

Bellaterra, Barcelona, Catalonia, Spain. Fax +34 935812790. Phone +34 93 5811303

E-mail address: itikawa@mat.uab.cat, jllibre@mat.uab.cat

2 Departamento de Matemática, Universidade Estadual de Campinas, Rua Sérgio Baruque de Holanda, 651, Cidade Universitária Zeferino Vaz, 13083-859, Campinas, SP, BRAZIL

E-mail address: ddnovaes@ime.unicamp.br 